\title{
Primary Burkitt's Lymphoma Presenting as a Rapidly Growing Thyroid Mass
}

\author{
Ibrahim Yildiz ${ }^{a}$ Fatma Sen $^{a} \quad$ Bahtiyar Toz $^{\mathrm{b}}$ Leyla Kilic $^{\mathrm{a}}$ \\ Mehmet Agan ${ }^{c}$ Mert Basaran ${ }^{a}$ \\ ${ }^{a}$ Department of Medical Oncology, Institute of Oncology, and Departments of \\ ${ }^{\mathrm{b}}$ Internal Medicine and ${ }^{\mathrm{C}}$ Pathology, Istanbul Faculty of Medicine, Istanbul University, \\ Istanbul, Turkey
}

\section{Key Words}

Burkitt's lymphoma $\cdot$ Chemotherapy $\cdot$ Thyroid

\begin{abstract}
A 31-year-old male patient presented with a rapidly growing neck mass with normal thyroid function tests. Ultrasonography showed thyroidal expansion, a hypoechoic nodule that completely filled the right lobe, and 2 hypoechoic lymphadenopathies in the right jugulodigastric chain. The patient underwent right total and left subtotal thyroidectomy, following the diagnosis of nodular goiter; however, postoperative histopathological evaluation demonstrated primary Burkitt's lymphoma of the thyroid gland. The tumor was staged as stage 1, and R-hyper-CVAD protocol (rituximab, hyperfractionated cyclophosphamide, vincristine, doxorubicin and dexamethasone) was administered. The protocol was changed to R-CHOP after 4 cycles due to recurrent grade III/IV cytopenias and febrile neutropenia. The PET-CT scans performed after chemotherapy and at the 6-month follow-up were normal. In summary, we reported a case with a diagnosis of Burkitt's lymphoma, which is a rare type of primary thyroid lymphoma.
\end{abstract}

\section{Introduction}

Primary lymphoma is an uncommon malignant disease of the thyroid gland. It accounts for $1-5 \%$ of all thyroid malignancies, $1-2.5 \%$ of all lymphomas, and $<3 \%$ of all extranodal non-Hodgkin's lymphomas [1-3]. Typically, it is more frequent in older women, and the female/male ratio varies from 1.4:1 to $6: 1[4,5]$. Normally, the thyroid gland does not contain lymphoid tissue; however, thyroid lymphomas generally occur in association with some immunologic disorders, such as Hashimoto's thyroiditis, or as 
a result of lymphoid tissue migration to the thyroid gland during inflammation [6, 7]. Here, we report a very unusual type of thyroid lymphoma.

\section{Case Report}

A 31-year-old male patient presented with a sore throat and a rapidly enlarging mass on the fore neck that began 5 days earlier. The patient did not report any pain localized in the swollen area. Additionally, he did not report any systemic symptoms, such as weight loss or fever. A $5 \times 4 \mathrm{~cm}$ nodule was palpated on the right lobe of the gland. Other systemic examinations were normal, without any palpable lymphadenopathy or organomegaly. Ultrasonography showed a heterogeneous/hypoechoic large nodule filling the right lobe, which was $4 \times 3.5 \times 3 \mathrm{~cm}$ in size. There were 2 hypoechoic lymphadenopathies $(15 \times 11 \mathrm{~mm})$ in the right jugulodigastric plexus, which did not contain fat tissue. Thyroid hormone test results were within normal ranges; TSH was $0.573 \mu \mathrm{IU} / \mathrm{ml}$ and anti-TPO was 29 $\mathrm{IU} / \mathrm{ml}$. Beta-2 microglobulin was $1.26 \mathrm{mg} / \mathrm{l}$ (normal range: 0-2.5), and EBV-VCA IgM, IgG, anti-HIV, HBsAg and anti-HCV were negative. The patient's blood count, serum biochemistry and erythrocyte sedimentation rate were within normal ranges.

The patient underwent right total and left subtotal thyroidectomy, following the initial diagnosis of nodular goiter. Histopathological examination of the frozen sections led to the diagnosis of lymphoma. The postoperative pathological examination was consistent with Burkitt's lymphoma in the right lobe of the thyroid gland and in 3 lymph nodes, Hashimoto's thyroiditis in the left lobe and reactive changes in 1 lymph node. High-power view (H\&E stain, $\times 1,000)$ revealed a monotonous medium-sized lymphoid cell infiltration with scant cytoplasm, small cytoplasmic vacuoles, a fine chromatin pattern and usually 2 or more nucleoli in the nucleus. Additionally, the immunohistochemical investigation showed widespread BCL-6 and CD10 immunoreactivity in CD20-positive and neoplastic cells; however, CD5, CD21, BCL2 and TdT were negative. The KI-67 proliferation index was $100 \%$ (fig. 1ac). Bone marrow examination was normal. An F-18 FDG PET-CT showed hypermetabolic lymph nodes (the largest was $1.7 \times 1 \mathrm{~cm}$ ), observed at level 3 of the right cervical region ( $\underline{\mathrm{fig} .2}$ ). According to these findings, stage 1 primary thyroid Burkitt's lymphoma was diagnosed.

R-Hyper-CVAD therapy (rituximab, hyperfractionated cyclophosphamide, vincristine, doxorubicin and dexamethasone) was administered. Complete response was achieved after 4 courses. However, the protocol was changed to R-CHOP after 4 cycles due to recurrent grade III/IV cytopenias and febrile neutropenia. The PET-CT scans performed after chemotherapy and at the 6-month follow-up were normal.

\section{Discussion}

Primary thyroid lymphoma (PTL) accounts for $2-4 \%$ of all thyroid malignancies and $<2 \%$ of all extranodal lymphomas. Most of these tumors are classified as B-cell lymphomas, which include mucosa-associated lymphoid tissue (MALT) lymphoma and diffuse large B-cell lymphoma (DLBCL) [3]. Recently, Watanabe et al. [8] clinically and pathologically reviewed 171 patients with PTL and investigated the clinical features and the treatment outcomes of PTL. The pathological diagnosis of PTL patients included DLBCL ( $\mathrm{n}=74)$, DLBCL with MALT lymphoma $(\mathrm{n}=13)$, MALT lymphoma $(\mathrm{n}=$ $80)$ and others $(n=4)$. In another study that reported the results of 108 PTL cases, no primary thyroid Burkitt's lymphoma was observed [9].

Hashimoto's thyroiditis and lymphoma of the thyroid co-exist in $40-80 \%$ of the patients. Hashimoto's thyroiditis induces reactive lymphoid proliferation leading to the development of MALT lymphoma $[6,7,10]$. MALT may eventually result in an aggressive lymphoma via accumulation of genetic abnormalities [11, 12]. Lymphomas originating in the thyroid frequently present with a rapidly enlarging mass in the fore 
neck, which causes nonspecific compression symptoms, including hoarseness, dysphagia, dyspnea and pressure sense. Nonetheless, upon presentation of a patient with a neck mass, physicians must consider lymphadenopathies, other types of thyroid and salivary gland neoplasms, thyroglossal cyst, laryngocele, teratoma, dermoid cyst, thymic cyst, ranula, paraganglioma, schwannoma and lipoma in the differential diagnosis.

The treatment of PTL generally depends on the histological subtype of the neoplasm, disease stage and tumor size as well as associated comorbidities. Chemotherapy alone is suggested for patients $<60$ years of age without any adverse prognostic factors; radiotherapy can be combined in selected cases [13]. Stage 1E MALT and other lowgrade thyroid lymphomas may be treated with regional irradiation alone because they generally remain localized at the primary site [3]. Other histopathological subtypes are rare, and therapeutic guidelines cannot be easily proposed. The role of surgery is principally only diagnostic. The results of the R-hyper-CVAD or R-CHOP chemotherapy regimens as treatment for primary thyroidal Burkitt's lymphoma have been reported only as case reports. In a review of 26 patients with PTL, $50 \%$ of the patients had DLBCL, 23\% had MALT lymphoma, 7\% had Hodgkin's lymphoma and in only 1 case Burkitt's lymphoma was observed. A 46-year-old male with Burkitt's lymphoma also had typical Hashimoto's thyroiditis with hypothyroidism and both the thyroid and the stomach were involved [14]. A 53-year-old male patient with Burkitt's lymphoma, who presented with a suddenly growing thyroid mass, was staged as stage IV disease due to bone involvement. The patient was given R-hyper-CVAD protocol and was reported as disease-free by PET-CT studies at the 6- and 27- month follow-up [15].

In conclusion, PTL should be suspected in a patient with a history of chronic lymphocytic thyroiditis presenting with a rapidly enlarging neck mass. Primary Burkitt's lymphoma of the thyroid is a very rare form of the disease and presents with diffuse B-cell lymphoma and MALT subtypes. Medical treatment is considered the principal therapeutic method for PTL, and surgical treatment is generally diagnostic. Immediate diagnosis and timely initiation of chemotherapy may provide a complete reponse with long progression-free survival.

\section{Disclosure Statement}

The authors have no conflicts of interest that are directly relevant to the content of this study. 

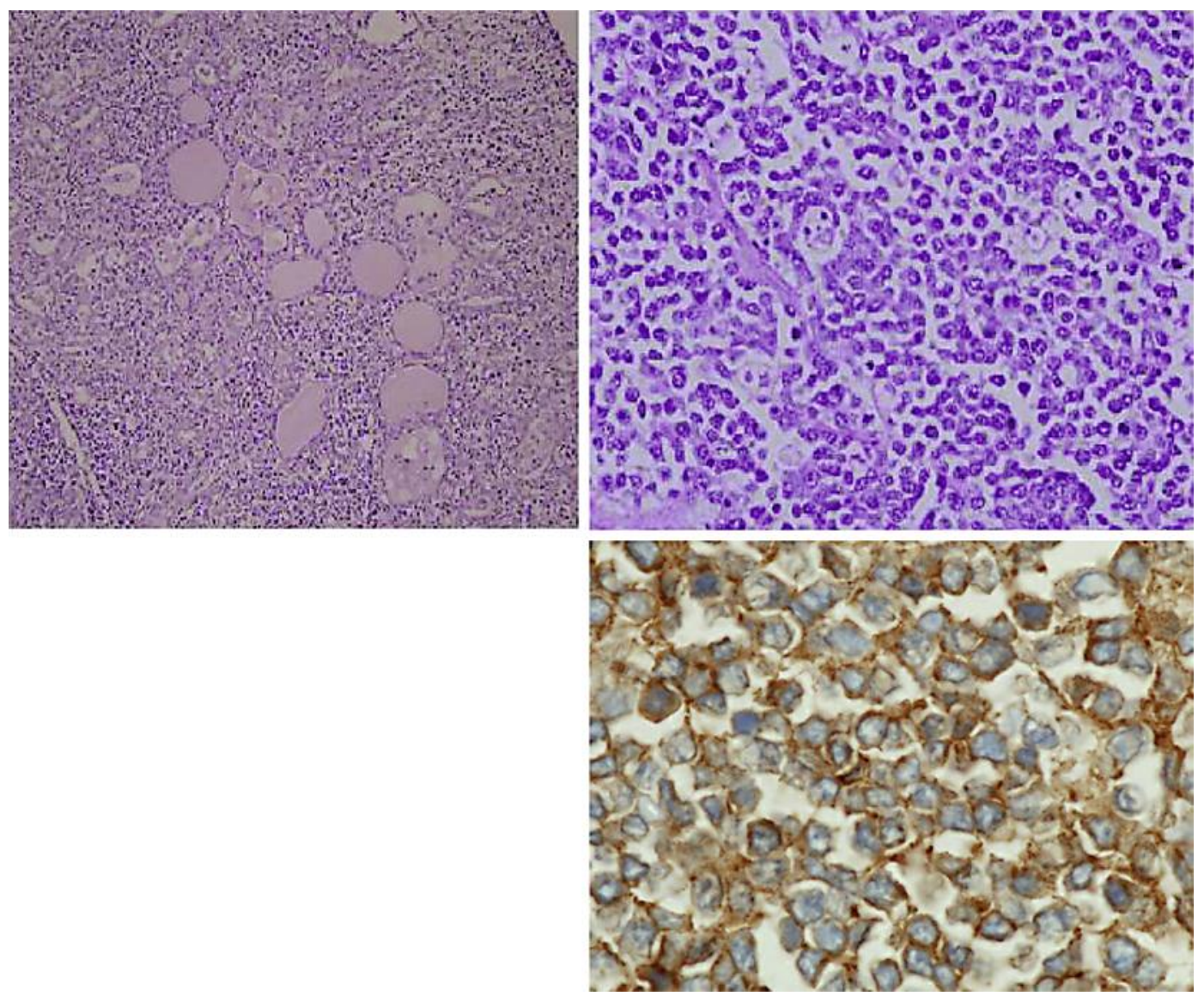

Fig. 1. Histopathological findings. There is a diffuse infiltrate of lymphoid cells in the thyroid gland (top left; H\&E stain, $\times 100$ ) with numerous mitoses and a prominent starry sky pattern because of the presence of multiple tangible body macrophages (top right; H\&E stain, $\times 1,000$ ). The neoplastic cells are $\mathrm{CD} 10+($ bottom; $\times 1,000)$. 


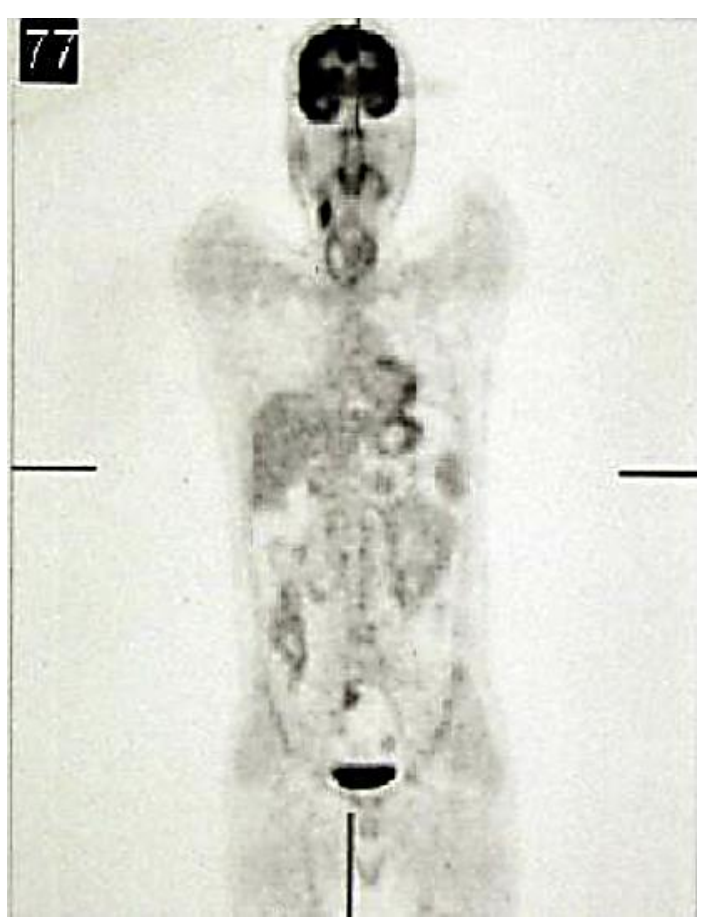

Fig. 2. The hypermetabolic lymph nodes are observed at level 3 of the right cervical region in the F-18 FDG PET-CT.

\section{References}

1 Ansell SM, Grant CS, Habermann TM: Primary thyroid lymphoma. Semin Oncol 1999;26:316-323.

2 Austin JR, el-Naggar AK, Goepfert H: Thyroid cancers. II. Medullary, anaplastic, lymphoma, sarcoma, squamous cell. Otolaryngol Clin North Am 1996;29:611-627.

-3 Pasieka JL: Anaplastic cancer, lymphoma, and metastases of the thyroid gland. Surg Oncol Clin North Am 1998;7:707-720.

4 Rasbach DA, Mondschein MS, Harris NL, Kaufman DS, Wang C: Malignant lymphoma of the thyroid gland: a clinical and pathologic study of twenty cases. Surgery 1985;98:1166-1170.

-5 Hamburger JI, Miller JM, Kini SR: Lymphoma of the thyroid. Ann Intern Med 1983;99:685-693.

-6 Kato I, Tajima K, Suchi T, Aozasa K, Matsuzuka F, Kuma K Tominaga S: Chronic thyroiditis as a risk factor of B-cell lymphoma in the thyroid gland. Jpn J Cancer Res 1985;76:1085-1090.

7 Holm L, Blomgren H, Lowhagen T: Cancer risks in patients with chronic lymphocytic thyroiditis. N Engl J Med 1985;312:601-604.

-8 Watanabe N, Noh JY, Narimatsu H, Takeuchi K, Yamaguchi T, Kameyama K, Kobayashi K, Kami M, Kubo A, Kunii Y, Shimizu T, Mukasa K, Otsuka F, Miyara A, Mina gawa A, Ito K, Ito K: Clinicopathological features of 171 cases of primary thyroid lymphoma: a long-term study involving 24553 patients with Hashimoto's disease. Br J Haematol. 2011;153;236-243.

9 Derringer GA, Thompson LD, Frommelt RA, Bijwaard KE, Heffess CS, Abbondanzo SL: Malignant lymphoma of the thyroid gland: a clinicopathologic study of 108 cases. American Journal of Surgical Pathology 2000;24:623-639.

10 Isaacson PG: The MALT lymphoma concept updated. Ann Oncol. 1995;6:319-320.

11 Peng H, Du M, Diss TC, Isaacson PG, Pan L: Genetic evidence for a clonal link between low and high-grade components in gastric MALT B-cell lymphoma. Histopathology 1997;30:425-429.

12 Chan JK, Ng CS, Isaacson PG: Relationship between high-grade lymphoma and low-grade B-cell mucosaassociated lymphoid tissue lymphoma (MALToma) of the stomach. Am J Pathol 1990;136:1153-1164. 
13 Miller TP, Dahlberg S, Cassady JR, Adelstein DJ, Spier CM, Grogan TM, LeBlanc M, Carlin S, Chase E, Fisher RI: Chemotherapy alone compared with chemotherapy plus radiotherapy for localized intermediateand high-grade non-Hodgkin's lymphoma. N Engl J Med 1998;339:21-26.

14 Thieblemont C, Mayer A, Dumontet C, Barbier Y, Callet-Bauchu E, Felman P, Berger F, Ducottet X, Martin C, Salles G, Orgiazzi J, Coiffier B: Primary thyroid lymphoma is a heterogeneous disease. Journal of Clinical Endocrinology and Metabolism 2002;87:105-111.

15 Kalinyak JE, Kong CS, McDougall IR: Burkitt lymphoma presenting as a rapidly growing thyroid mass. Thyroid 2006;16:1053-1057. 